\title{
VLBI studies of the nutations of the earth
}

\author{
T. A. Herring, Harvard-Smithsonian Center for Astrophysics \\ 60 Garden Street \\ Cambridge, MA. 02138
}

\begin{abstract}
The application of very-long-baseline interferometry (VLBI) to the study of the nutations of the earth has yielded unprecedented accuracy for the experimental determination of the coefficients of the nutation series. The analysis of six years of VLBI data has yielded corrections to the coefficients of the seven largest terms in the IAU 1980 nutation series with periods of one year or less, with accuracies approaching the truncation error of this nutation series $(0.1$ mas). The nutation series coefficients computed from the VLBI data, and those obtained from theoretical considerations (the IAU 1980 nutation series), are in excellent agreement. The largest corrections are to the coefficients of the retrograde annual nutation $[2.0 \pm 0.1 \mathrm{mas}]$, the prograde semiannual nutation $[(0.5-\imath 0.4)$ $\pm 0.1 \mathrm{mas}]$, and the prograde 13.7 day nutation $[-0.4 \pm 0.1 \mathrm{mas}]$. (The imaginary term for the semiannual nutation represents a term $90^{\circ}$ out-of-phase with the arguments of the nutation series.) The geophysical implications of these results are currently under active investigation. We discuss the methods used to extract the nutation information from the VLBI data, the calculations of the uncertainties of the resultant corrections to the coefficients of the nutation series, and the current research into the nutations of the earth.
\end{abstract}

\section{INTRODUCTION}

The determination of the coefficients of the nutation series using very-longbaseline interferometry data has been discussed recently in a number of papers (Herring et al., 1983; Gwinn et al., 1984; Herring et al., 1985; Eubanks et al., 1985; Gwinn et al., 1986; Herring et al., 1986a; Herring et al., 1986b; and Himwich et al., 1986). A detailed description of the analysis techniques used for determining the corrections to the coefficients of the nutation series is given in Herring et al., 1986a. The interpretation of the results given in Herring et al., 1986a is discussed in detail by Gwinn et al., 1986. The interpretation of these results has also been discussed by Wahr and Bergen et al., 1986; Yoder and Ivinus, 1986; and Yoder and Ivinus, 1987. Here we review briefly the procedures used to estimate the corrections to the coefficients of the nutations, and we present the results obtained from the analysis of 412 VLBI experiments obtained during the 6.5 year period between July 1980 and February 1987. 


\section{DATA ANALYSIS TECHNIQUE}

The techniques we have used to estimate corrections to the IAU 1980 nutation series have been discussed in Herring et al.(1985) and Herring et al.(1986a). For each VLBI observing session (typically of 24 hours duration), we estimate corrections, $\delta \Delta \psi$ and $\delta \Delta \epsilon$, to the nutation angles computed from the IAU 1980 nutation series. In a post-processing operation, we use these nutation-angle corrections to estimate corrections to the coefficients of certain terms in the nutation series. An alternative technique is to directly estimate the corrections to coefficients of the nutation series from the VLBI data themselves. Results obtained using this technique have been presented by Himwich et al., 1986.

The nutation-angle corrections are not only used to estimate the corrections to the coefficients of the nutation series, but they are also be used to study the noise characteristics of the nutation estimates (see Herring et al.,1986a), and to study phenomena not explicitly included in the standard nutation series such as the "free core-nutation" (see Herring et al., 1986b). In particular, we will discuss the power-spectral density (PSD) function of the nutation-angle corrections. The calculation of the PSD function from the nutation-angle corrections have been discussed in Herring et al., 1985. The technique we adopt is to "lightly" smooth the nutation-angle corrections using a Gaussian filter with a full-width-at-halfmaximum (FWHM) of 5 days, and to then compute the PSD function from these uniformly spaced smoothed values (5 day spacing). The (assumed uncorrelated) noise in the nutation-angle corrections is propagated through the smoothing and the fast fourier transform (FFT) algorithms to compute the $99.5 \%$ confidence limit for the PSD function.

\section{RESULTS}

The VLBI data we have analyzed are from 412 observing sessions carried out by the NASA Crustal Dynamics Project and the NGS IRIS program between July 1980 and February 1987. This data set is an extension of those discussed in Herring et al.(1985) and Herring et al.(1986a and b). In Table 1, we give the estimates of the amplitudes of the circular nutations that can be resolved with the limited temporal range of the VLBI data. The values for $\delta \Delta \epsilon$ and $\delta \Delta \psi \sin \epsilon_{\circ}$ we used to obtain these amplitude estimates are shown in Figure 1. The PSD function computed from the nutation-angle corrections shown in Figure 1 is shown in Figure 2. The three largest peaks in the PSD function correspond to the three largest corrections to the coefficients of the nutation series given in Table 1 . There is also a large peak corresponding to the retrograde frequency closest to 1 cycle per sidereal day. Any corrections to the long period nutations (18.6 and 9.3 years) would all appear in this term. With only 6.5 years of data we can not yet reliably estimate corrections to the nutation series terms with these long periods. The remainder of the PSD function appears to be consistent with the white-noise statistics used to compute the $99.5 \%$ confidence interval.

The results given in Table 1 are very consistent with previously published results within the uncertainties of these earlier analyses. The correction to the retrograde annual nutation has been interpreted as being due to the effects of the earth's fluid core being more strongly coupled to the mantle than was inferred for the calculation of the IAU 1980 nutation series (Gwinn et al., 1986). The corrections to the prograde semiannual term are partly due the coupling of the fluid core to the mantle and partly due to the effects of ocean tides on the nutations (Sasao and Wahr, 1981). The correction to the 13.7 day prograde term has not 
TABLE 1. Estimates of Corrections to Amplitudes of Circular Nutations of IAU 1980 Nutation Series (Wahr, 1981).

\begin{tabular}{cccc} 
& & \multicolumn{2}{c}{ Corrections $^{*}$} \\
\cline { 3 - 4 } $\begin{array}{c}\text { Period } \\
\text { in inertial }\end{array}$ & $\begin{array}{c}\text { Observed } \\
\text { smplitude, }\end{array}$ & $\begin{array}{c}\text { In-Phase } \\
\delta a_{r}, \\
\text { mas }\end{array}$ & $\begin{array}{c}\text { Out-of-Phase } \\
\delta a_{i}, \\
\text { mas }\end{array}$ \\
\hline 182.6 prograde & -548.62 & 0.45 & -0.41 \\
182.6 retrograde & -24.63 & -0.11 & -0.07 \\
13.7 prograde & -94.45 & -0.37 & -0.00 \\
13.7 retrograde & -3.63 & -0.03 & 0.05 \\
365.3 prograde & 25.71 & 0.05 & 0.18 \\
365.3 retrograde & -33.13 & -2.07 & 0.36 \\
27.6 prograde & 14.50 & -0.02 & 0.06 \\
27.6 retrograde & -13.79 & 0.03 & -0.01 \\
121.7 prograde & -21.56 & -0.08 & -0.03 \\
121.7 retrograde & -0.95 & -0.04 & -0.03 \\
9.1 prograde & -12.53 & -0.10 & 0.00 \\
9.1 retrograde & -0.43 & -0.01 & -0.02 \\
31.8 prograde & 3.19 & -0.01 & -0.03 \\
31.8 retrograde & -3.02 & 0.07 & 0.00 \\
433.2 prograde & $0.06 \dagger$ & -0.06 & -0.00 \\
433.2 retrograde FCN & $0.33 \dagger$ & 0.32 & -0.04 \\
\hline
\end{tabular}

*The standard deviation of each correction is estimated to be 0.10 mas. The standard deviations are obtained using the techniques described in Herring et al. (1986), with the root-sum-squares addition of a contribution of 0.07 mas to account for (i) the effects of the truncation of the IAU 1980 nutation series to the nearest 0.1 mas, (ii) the possible effects of atmospheric excitation of the nutation, and (iii) the effects of ocean and earth tide modeling errors in the analysis of the VLBI data. See also Herring et al. (1986) for definitions of the quantities listed.

†The amplitudes of these terms are the root-sum-squares of the amplitudes of the real and imaginary components.

been satisfactorily explained. Since this nutation term is the one most affected by the elasticity of the earth, the correction may arise from the treatment of elasticity or the neglect of the effects of anelasticity in the IAU nutation series. The rootmean-square scatter of the remaining 25 corrections given in Table 1 is 0.06 mas, which is consistent with our estimated uncertainties of 0.10 mas.

\section{CONCLUSION}

The quality of the VLBI estimates of the corrections to the nutation series will improve as further VLBI experiments are carried out. The VLBI data from the international earth rotation service will continue to provide high quality measurements of the nutation angles on a regular basis (once every 5 days). These data and those obtained by the NASA Crustal Dynamics Project will ensure that the coefficients of selected terms in the nutation series will be determined with precisions of less than $\mathbf{0 . 1}$ mas. However, the current nutation series is truncated at 0.1 mas, and thus, will soon be the limiting error source in the interpretation of the corrections to the nutation series. Since all modern nutation series are obtained by convolving the response function of a "realistic" model earth with the nutations of a rigid earth, we will soon start developing a rigid-earth nutation 


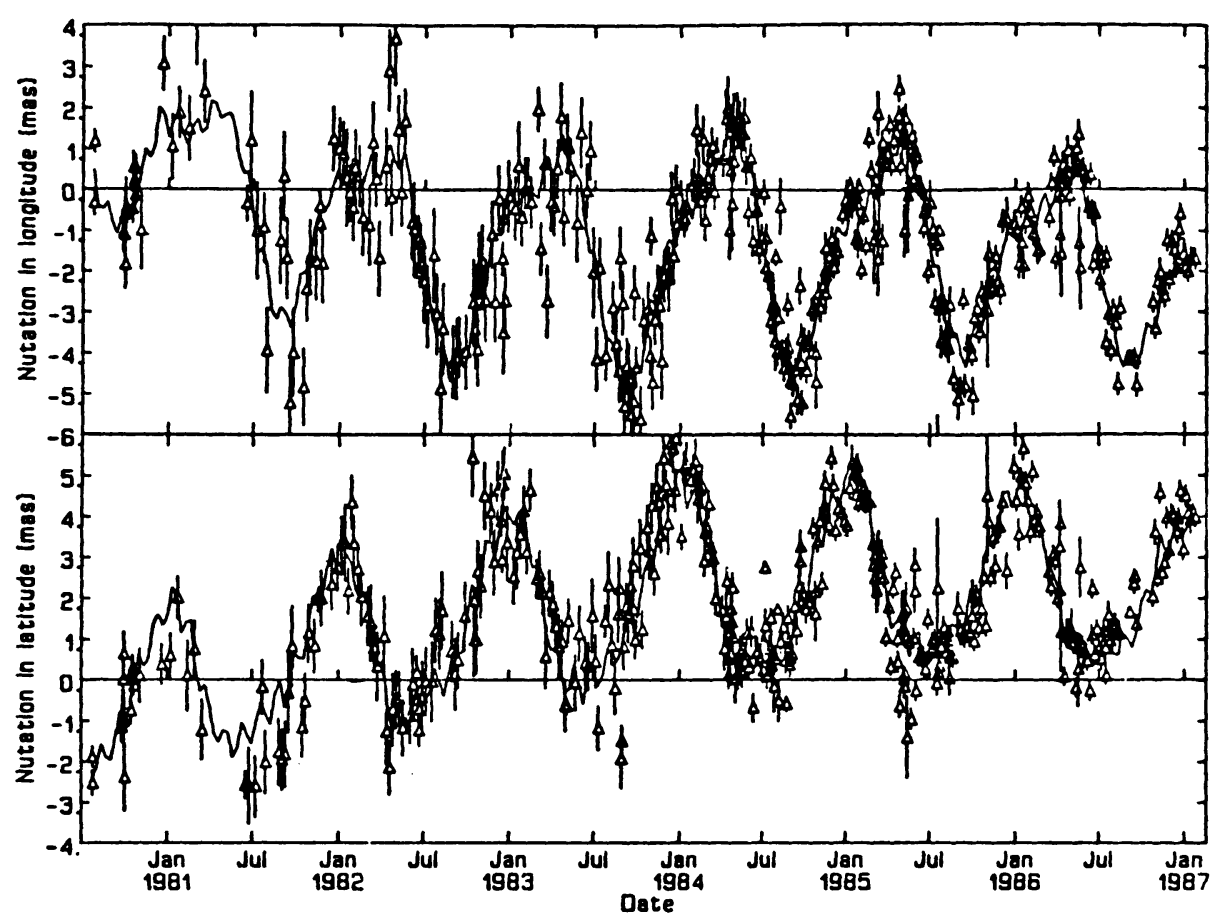

Figure 1. Estimates of $\delta \Delta \epsilon$ and $\delta \Delta \psi \sin \epsilon_{\circ}$ from the VLBI data analyzed in this paper. The solid line in each figure is computed from the corrections given in Table 1 and from long-period terms (periods $\geq 18.6$ years) which are not given in the table because their values are highly correlated and the estimates are not considered reliable.

series with an accuracy of 0.01 mas. This new nutation series will provide an accurate framework in which the observed nutations of the earth can be compared with that computed from geophysical theory.

\section{ACKNOWLEDGMENTS}

This work was supported by the Air Force Geophysics Laboratory, contract F-19628-86-K-0025; NASA grant NAG5-538; and NSF grants EAR-83-06380 and EAR-86-18989.

\section{REFERENCES}

Eubanks, T.M. et al., in "Proc. of the international conference on earth rotation and the terrestrial reference frame", Ohio State University, 326-340, 1985.

Gwinn, C.R. et al., EOS, 65, 859, 1984.

Gwinn, C.R. et al., J. Geophys. Res., 91, 4755-4765, 1986.

Herring, T.A. et al., EOS, 64, 674, 1983.

Herring, T.A. et al., in "Proc. of the international conference on earth rotation and the terrestrial reference frame", Ohio State University, 307-325, 1985. 


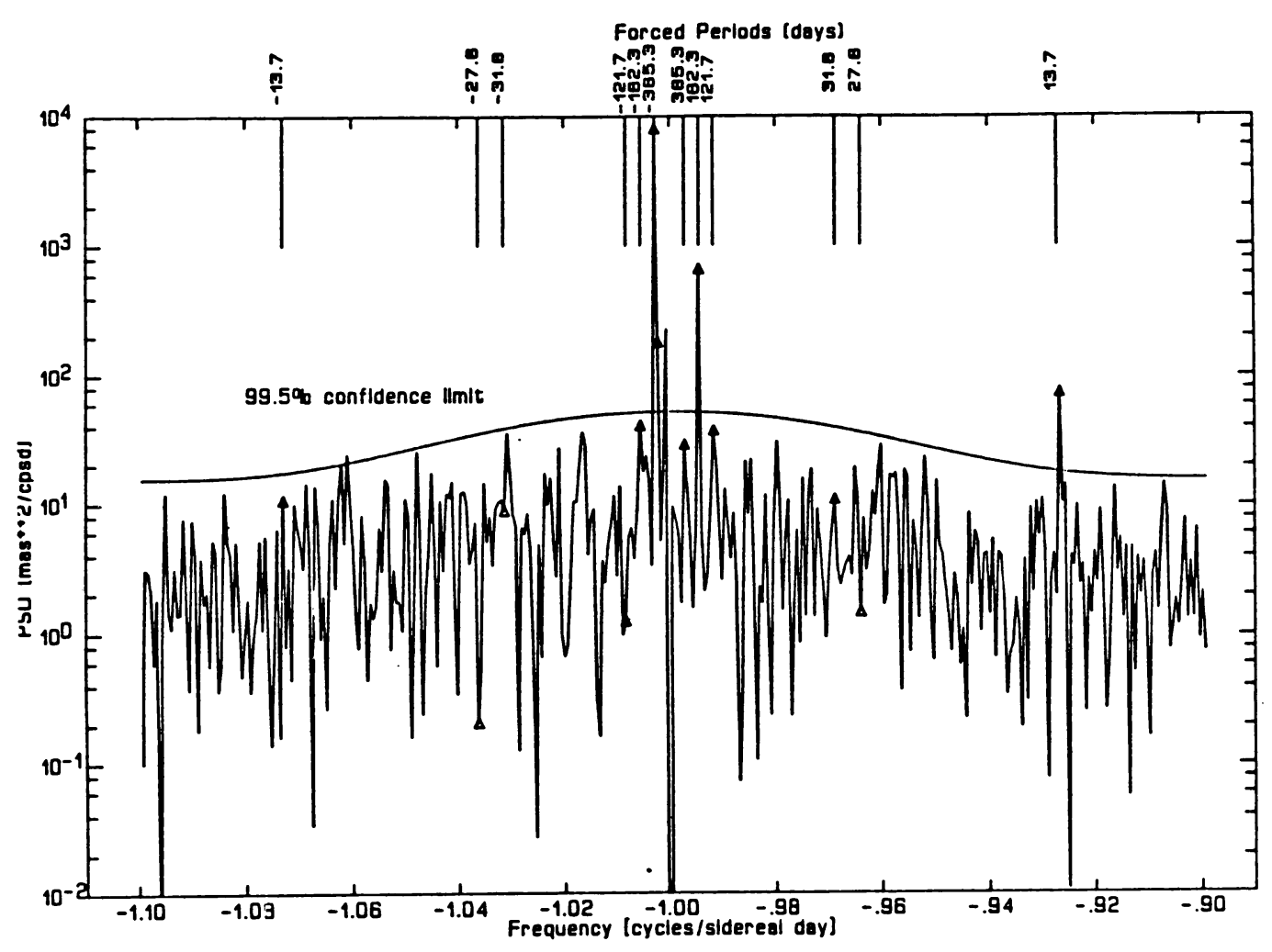

Figure 2. The power-spectral-density (PSD) function of the nutation-angle corrections shown in Figure 1. The decrease in power in the PSD function and in the $99.5 \%$ confidence limit with increasing frequency away from -1 cycle per sidereal day (cpsd) is due to use of a (5 day FWHM) Gaussian filter. The frequency spacing in the PSD function is $1 / 1830 \mathrm{cpsd}$. At the top of the figure we have shown the frequency positions of some of the major terms in the nutation series. We have also marked the frequency components in the PSD function closest to these frequencies with triangles.

Herring, T.A. et al., J. Geophys. Res., 91, 4745-4754, 1986a.

Herring, T.A. et al., in "Proc. of IAU Symp. No. 128", $1986 \mathrm{~b}$.

Himwich, W.E. et al., in "Proc. of IAU Symp. No. 128", 1986.

Sasao, T. and J.M.Wahr, Geophys. J. Roy. Astr. Soc., 64, 729-746, 1981.

Wahr, J.M. and T.Sasao, Geophys. J. Roy. Astr. Soc., 64, 747-765, 1981.

Wahr, J.M. and Z. Bergen, Geophys. J. R. Astr. Soc., 87, 633-668, 1986.

Yoder, C.F. and E.R. Ivinis, in "Proc. of IAU Symp. No. 128", 1986.

Yoder, C.F. and E.R. Ivinis, in "Proc. of IAU Symp. No. 129", 1987. 